\title{
EDITORIAL
}

\section{Editorial: Schweizerische Gesellschaft für Sportpsychiatrie und -psychotherapie SGSPP}

\author{
EXERCISE IS MEDICINE
}

\section{Claussen Malte Christian}

Präsident und Ressortleiter Erwachsenenpsychiatrie und -psychotherapie, Schweizerische Gesellschaft für Sportpsychiatrie und -psychotherapie SGSPP

Ärztlicher Leiter Sportpsychiatrie und -psychotherapie, Klinik für Psychiatrie, Psychotherapie und Psychosomatik, Psychiatrische Universitätsklinik Zürich, Privatklinik Wyss AG und Psychiatrische Dienste Graubünden

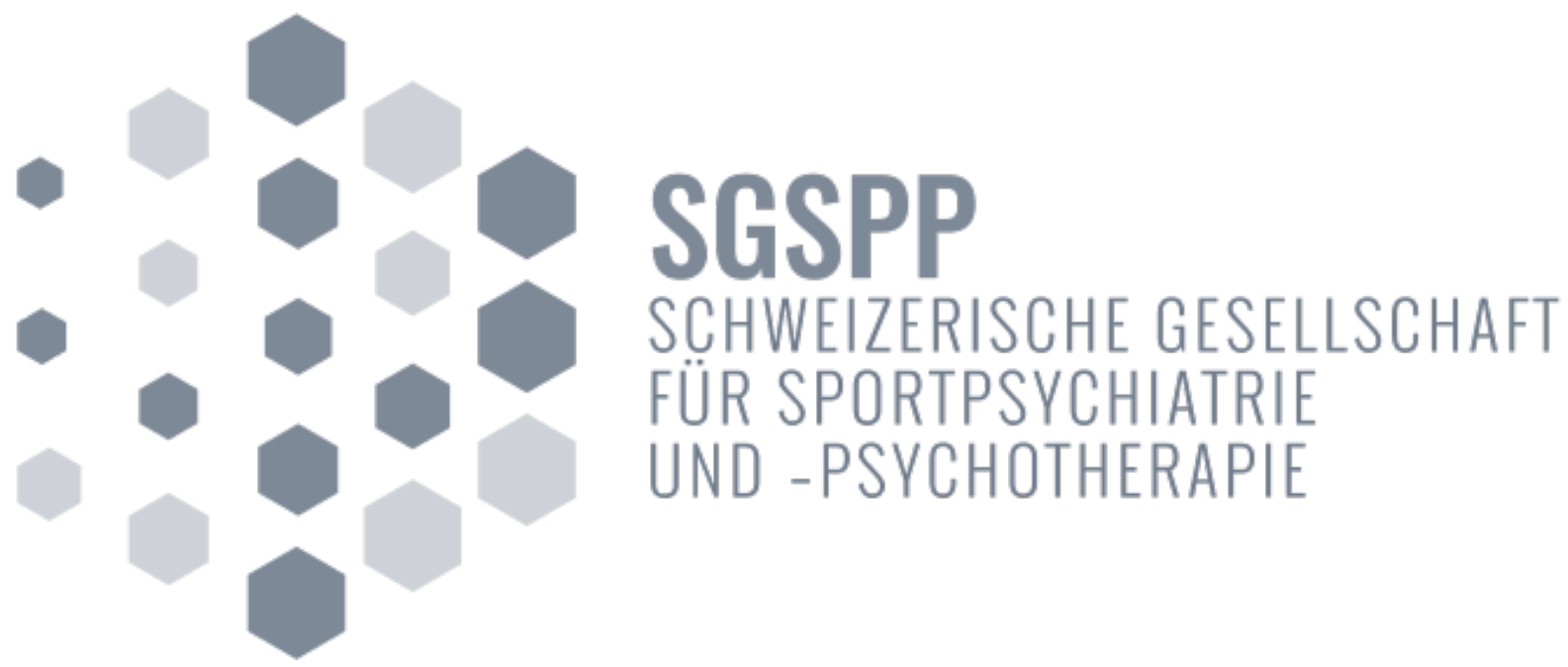

Die Schweizerische Gesellschaft für Sportpsychiatrie und -psychotherapie SGSPP wurde am 29. März 2019 in den Räumen der Privatklinik Wyss AG in Münchenbuchsee, Kanton Bern gegründet. Sie ist weltweit, nach der International Society of Sports Psychiatry ISSP, die zweite sportpsychiatrische und psychotherapeutische Fachgesellschaft und zudem die erste nationale Fachgesellschaft dieser Art. Als Zweck der SGSPP wurde die Förderung der Sportpsychiatrie und -psychotherapie über die Lebensspanne in der Schweiz, im Leistungssport und in der Allgemeinbevölkerung formuliert. Hieraus ergeben sich zwei Tätigkeitsfelder der SGSPP: Psychische Gesundheit im Leistungssport; Sport, Training und Bewegung in Prävention und Therapie psychischer Erkrankungen. Über die Lebensspanne bedeutet, dass die SGSPP die 
Sportpsychiatrie und -psychotherapie gleichermassen für Kinder und Jugendliche, Erwachsene und Ältere fördern möchte. Dies bildet sich auch im SGSPP-Vorstand ab, so gibt es neben dem Ressort Forschung und Lehre, ein Ressort Kinder- und Jugendpsychiatrie und -psychotherapie, Erwachsenenpsychiatrie und psychotherapie und Alterspsychiatrie und -psychotherapie.

Die SGSPP versteht sich als sportpsychiatrische und

-psychotherapeutische Fachgesellschaft, die sich als Teilgebiet der Psychiatrie und Psychotherapie und der Kinder- und Jugendpsychiatrie und -psychotherapie definiert und sich zugleich - aufgrund der besonderen und interdisziplinären Thematik - auch als Teilgebiet des Querschnittsfachs Sportmedizin entwickeln möchte. Der Aufbau eines sportmedizinischen-sportpsychiatrischen Austauschgefässes und der Austausch in der medizinischen Aus-, Weiter- und Fortbildung mit Sport and Exercise Medicine Switzerland (SEMS) wären aus Sicht der SGSPP geeignete, erste Anknüpfungspunkte. Die SGSPP ist bereit, hierzu einen substanziellen Beitrag zu leisten.

In ihrem Verständnis dient die SGSPP als Interessenvertretung für Sportpsychiater und psychotherapeuten und zielt gleichermassen auf die interdisziplinäre Einbindung anderer Berufsgruppen, um die Entwicklung der Sportpsychiatrie und -psychotherapie zu fördern. Zu den Kernaktivitäten der SGSPP gehören der interdisziplinäre Austausch zum Beispiel mit der Sportmedizin, den Sportwissenschaften und der Sportpsychologie, wie der Brückenschlag zur klinischen Psychiatrie und Psychotherapie. Dies geschieht durch eine Vernetzung mit den genannten Fachdisziplinen und ihren Gesellschaften sowie durch Aufklärung und Publikationen. Darüber hinaus versteht sich die SGSPP als eine Fachgesellschaft, die Forschungs- und Lehraktivitäten unterstützt und Aufklärungsarbeit zur Prävention psychischer Erkrankungen leistet und sich für die Entstigmatisierung psychischer Leiden engagiert. In Kurzbeiträgen zu verschiedenen sportpsychiatrischen und -psychotherapeutischen Themen soll in dieser Ausgabe Sport \& Exercise Medicine Switzerland ein Einblick in die (geplanten) Forschungsinitiativen gegeben werden. Diese Initiativen erfolgen bereits heute oft schon interdisziplinär und klinik-, instituts- und praxisübergreifend. Die SGSPP fördert diesen Austausch, so ermöglichen zum Beispiel die SGSPP-Homepage und -Veranstaltungen, Forschenden als Arbeitsgruppe sichtbar zu werden. Positionspapiere zu beiden Tätigkeitsfeldern der SGSPP wurden in den letzten Monaten durch den Vorstand der SGSPP erarbeitet und werden in englischer Sprache in dieser Ausgabe wiedergegeben. In den Positionspapieren werden zuerst die Problemfelder beschrieben und dann mögliche Ansatzpunkte und Empfehlungen hergeleitet. Ein weiterer Diskurs der Positionspapiere ist gewünscht und überdies notwendig, für eine Verbesserung der psychischen Gesundheit im Leistungssport, respektive einer besseren Integration von Sport, Training und Bewegung in den Präventions- und Therapiekonzepten psychischer Erkrankungen.

Zentral für jede Fachdisziplin ist eine systematische Wissensvermittlung. Ein dreistufiges Curriculum für die Qualifikation in Sportpsychiatrie und -psychotherapie wurde in den letzten Monaten durch eine Arbeitsgruppe der SGSPP mit der Deutschen Gesellschaft für Sportpsychiatrie und -psychotherapie DGSPP entwickelt. Dieses Curriculum ist das erste seiner Art. Die Stufe 1 «Sportpsychiatrische und psychotherapeutische Basisversorgung» soll Psychiatern und Psychotherapeuten, Kinder- und Jugendpsychiatern und -psychotherapeuten, psychologischen Psychotherapeuten und Sportmedizinern einen Einblick in das Gebiet geben und Grundkenntnisse vermitteln. Die Stufe 2 «Klinisch praktische Sportpsychiatrie und -psychotherapie» zielt auf den klinischen Einsatz von Sport, Training und Bewegung ab, während die darauf aufbauende 3. Stufe den Fokus auf den Leistungssport richtet und zu einem 
Fähigkeitsausweis «Sportpsychiatrie und -psychotherapie» der SGSPP führen soll. Im letzten Beitrag zur Sportpsychiatrie und -psychotherapie wird das SGSPP Curriculum Sportpsychiatrie und -psychotherapie vorgestellt.

Um der dynamischen Entwicklung der Sportpsychiatrie und -psychotherapie der Gegenwart Rechnung zu tragen, hat die SGSPP mit inländischen wie auch internationalen Fachgesellschaften und Fachreferaten Kooperationsvereinbarungen getroffen. Dies soll dazu dienen, neue Entwicklungen und Erkenntnisse im Bereich Sport und Psyche rascher und effektiver auszutauschen und das Gebiet der Sportpsychiatrie und psychotherapie weiter zu etablieren. Neben dem persönlichen Austausch sind vor allem gemeinsame Veranstaltungen, Symposien und Kongresse sowie Publikationen geeignete Möglichkeiten, um die angestrebten Ziele verwirklichen zu können. Die SGSPP bekennt sich zur interdisziplinären und auch internationalen Zusammenarbeit.

Eine aktive Teilnahme am weiteren Aufbau der Schweizerischen Gesellschaft für Sportpsychiatrie und psychotherapie ist sehr willkommen. Die 2. SGSPP-Jahrestagung findet vom 22. bis 23.1.2021 in der Psychiatrischen Universitätsklinik Zürich statt, zu der wir Sie herzlich einladen. Informationen zu Mitgliedschaft und Jahrestagung, aktuelle Nachrichten und weitere Informationen zur SGSPP und der Sportpsychiatrie und -psychotherapie finden sich auf http://www.sgspp.ch.

\section{Korrespondenzadresse}

Dr. med. Malte Christian Claussen

Präsident SGSPP

c/o Sportpsychiatrie und -psychotherapie,

Klinik für Psychiatrie, Psychotherapie

und Psychosomatik, Psychiatrische Universitätsklinik Zürich

Lenggstrasse 31, CH-8032 Zürich

E-Mail: malte.claussen@pukzh.ch

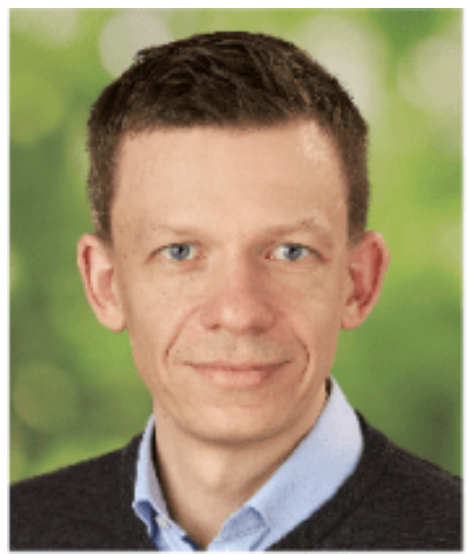

